\title{
Nonminimal Lorentz violation
}

\author{
Matthew Mewes \\ Physics Department, California Polytechnic State University \\ San Luis Obispo, CA 93407, USA
}

\begin{abstract}
This contribution to the CPT'16 meeting provides a brief overview of recent studies of nonminimal Lorentz violation in the Standard-Model Extension.
\end{abstract}

The Standard-Model Extension (SME) provides a general field-theoretic description of violations of Lorentz and CPT invariance in particle physics and in gravity and has facilitated hundreds of experimental tests of these fundamental symmetries. ${ }^{1}$ A Lorentz-violating term in the lagrangian of the SME is constructed from a tensor coefficient contracted with a conventional tensor operator, giving contributions to the action that schematically take the form

$$
\delta S=\int d^{4} x \text { (coefficient tensor) } \cdot(\text { tensor operator }) .
$$

The coefficients for Lorentz violation give the vacuum a non-scalar structure and act as Lorentz-violating background fields. The various violations are often classified according to the mass dimension $d$ of the associated operator.

Early development of the SME focused largely on minimal violations those involving Lorentz-violating operators with renormalizable mass dimensions $d=3$ and 4 - leading to the so-called minimal SME (mSME). Pioneering works constructed the minimal modifications to the Standard Model of particle physics ${ }^{2}$ followed by General Relativity. ${ }^{3}$ While the mSME has served as the theoretical basis for a vast majority of Lorentz tests performed to date ${ }^{1}$ more recent efforts aim at exploring nonminimal violations associated with operators of mass dimensions $d \geq 5$. These include the construction of the full nonminimal extensions for free photons, ${ }^{4}$ neutrinos, ${ }^{5}$ free Dirac fermions, ${ }^{6}$ and linearized gravity. ${ }^{7-9}$

The study of Lorentz-violating operators of nonrenormalizable dimensions can be motivated through simple dimensional analysis. For operators 
of dimension $d$, the coefficients for Lorentz violation have mass dimension $4-d$. Assuming a connection to Planck-scale physics, we might naively expect coefficients that are of order $\sim M_{P}^{4-d}$, where $M_{P}$ is the Planck mass. This type of suppression gives the SME the structure of a series approximation, which fits well with the notion that it represents the low-energy limit of some underlying high-energy theory. The idea is that conventional Lorentzinvariant physics (the Standard Model and General Relativity) corresponds to the dominant leading-order terms in the series. The Lorentz-violating terms in the SME give higher-order corrections, which decrease in size with higher dimension $d$. This makes sense for nonminimal violations with $d \geq 5$, but is problematic for minimal violations, where $d=4$ violations would be unsuppressed and $d=3$ violations would be very large. Minimal violations would be easily detected in this picture and precluded by their absence. The lowest-order Lorentz violations would then be nonminimal ones with mass dimension $d \geq 5$.

While the mSME has been tested extensively, the nonminimal parts of the SME remain relatively unexplored, with most of the parameter space unconstrained and completely open to future experimentation. At present, constraints on nonminimal Lorentz violation in photons include tests involving vacuum birefringence, ${ }^{4,10}$ vacuum dispersion, ${ }^{11}$ and resonant cavities. ${ }^{12}$ In neutrinos, there are constraints from oscillation experiments ${ }^{5}$ and kinematical tests involving high-energy astrophysical neutrinos. ${ }^{5,13}$ For heavier fermions, current constraints on nonminimal violations come from studies of muonic atoms ${ }^{14}$ and hydrogen-like atoms. ${ }^{15}$ Bounds on nonminimal Lorentz violation in gravity have been obtained in tests of short-range gravity, ${ }^{7,16}$ from limits on gravitational Čerenkov radiation in cosmic rays, ${ }^{8}$ and from limits on birefringence in gravitational waves. ${ }^{9}$

The different sectors of the nonminimal SME share some common features, which we illustrate here by considering the recently constructed nonminimal extension for linearized General Relativity. ${ }^{9}$ The lagrangian for this extension, in term of the metric perturbation $h_{\mu \nu}$, can be written

$$
\begin{aligned}
\mathcal{L}= & \frac{1}{4} \epsilon^{\mu \rho \alpha \kappa} \epsilon^{\nu \sigma \beta \lambda} \eta_{\kappa \lambda} h_{\mu \nu} \partial_{\alpha} \partial_{\beta} h_{\rho \sigma} \\
& +\frac{1}{4} h_{\mu \nu} \widehat{s}^{\mu \nu \rho \sigma} h_{\rho \sigma}+\frac{1}{4} h_{\mu \nu} \widehat{q}^{\mu \nu \rho \sigma} h_{\rho \sigma}+\frac{1}{4} h_{\mu \nu} \widehat{k}^{\mu \nu \rho \sigma} h_{\rho \sigma},
\end{aligned}
$$

where the first term is the usual Lorentz-invariant part. The term involving the tensor operator $\widehat{s}^{\mu \nu \rho \sigma}$ contains CPT-even Lorentz violations for even $d \geq 4, \widehat{q}^{\mu \nu \rho \sigma}$ gives CPT-odd violations with odd $d \geq 5$, and $\widehat{k}^{\mu \nu \rho \sigma}$ gives CPT-even violations with even $d \geq 6$. Each of the tensor operators represents an infinite series of derivatives. For example, $\widehat{s}^{\mu \rho \nu \sigma}$ is given by the 
expansion $\widehat{s}^{\mu \rho \nu \sigma}=\sum_{d} s^{(d) \mu \rho \epsilon_{1} \nu \sigma \epsilon_{2} \epsilon_{3} \ldots \epsilon_{d-2}} \partial_{\epsilon_{1}} \partial_{\epsilon_{2}} \partial_{\epsilon_{3}} \ldots \partial_{\epsilon_{d-2}}$. The expansion coefficients $s^{(d) \mu \rho \epsilon_{1} \nu \sigma \epsilon_{2} \epsilon_{3} \ldots \epsilon_{d-2}}$ are constant and control the Lorentz violation. The differing symmetries of the coefficients in each class result in different physical consequences.

The minimal version of the above theory is obtained by restricting attention to $d=4 \widehat{s}^{\mu \nu \rho \sigma}$ terms, which has the effect of demoting the operator $\widehat{s}^{\mu \nu \rho \sigma}$ to the constant $s^{(4) \mu \nu \rho \sigma}$. In other sectors of the SME, going the other way, from the minimal to the nonminimal extension, is effectively the reverse of this procedure. In almost every case, the introduction of nonminimal violations can be viewed as the promotion of minimal $d=3$, 4 coefficients from constants to operators. ${ }^{4-6}$ The exception is linearized General Relativity, where the operators $\widehat{q}^{\mu \nu \rho \sigma}$ and $\widehat{k}^{\mu \nu \rho \sigma}$ lack minimal counterparts.

The power of the SME lies in its ability to give theoretically consistent predictions for almost any system. For example, with the theory in Eq. (2) we can examine the effects of general Lorentz violation on gravitational waves. A key result is the modified dispersion relation, $\omega=\left(1-\varsigma^{0} \pm \sqrt{\left(\varsigma^{1}\right)^{2}+\left(\varsigma^{2}\right)^{2}+\left(\varsigma^{3}\right)^{2}}\right)|\boldsymbol{p}|$, which predicts several unconventional features, including dispersion and birefringence. The $\varsigma^{a}$ parameters are complicated momentum-dependent combinations of the coefficients for Lorentz violation. However, as in many other practical applications of the SME, the problem can be made tractable by performing a sphericalharmonic decomposition. This aids in the cataloging of the many Lorentzviolating effects that can arise. It also significantly simplifies the rotations between noninertial laboratory frames and the inertial Sun-centered frame conventionally used for reporting results. ${ }^{17}$

In the current example, the spherical expansion of the $\varsigma^{a}$ parameters is

$$
\begin{gathered}
\varsigma^{0}=\sum \omega^{d-4} Y_{j m}(-\hat{\boldsymbol{p}}) k_{(I) j m}^{(d)}, \quad \varsigma^{3}=\sum_{d j m} \omega^{d-4} Y_{j m}(-\hat{\boldsymbol{p}}) k_{(V) j m}^{(d)}, \\
\varsigma^{1} \mp i \varsigma^{2}=\sum \omega^{d-4}{ }_{ \pm 4} Y_{j m}(-\hat{\boldsymbol{p}})\left(k_{(E) j m}^{(d)} \pm i k_{(B) j m}^{(d)}\right) .
\end{gathered}
$$

The spherical coefficients for Lorentz violation $k_{(I) j m}^{(d)}, k_{(E) j m}^{(d)}, k_{(B) j m}^{(d)}$, and $k_{(V) j m}^{(d)}$ completely characterize the leading-order effects of Lorentz violation in gravitational waves. Currently, the $k_{(I) j m}^{(d)}$ coefficients have been bounded by the apparent absence of gravitational Čerenkov radiation in cosmic rays. ${ }^{8}$ The $k_{(E) j m}^{(d)}, k_{(B) j m}^{(d)}$, and $k_{(V) j m}^{(d)}$ coefficients are constrained by limits on birefringence in gravitational waves. ${ }^{9}$

Note that the above expansion involves spin-weighted spherical harmonics ${ }_{s} Y_{j m}$. These are similar to the familiar harmonics $Y_{j m}={ }_{0} Y_{j m}$, 
but carry spin weight $s$, which is equivalent to helicity, up to a sign. The appearance of the $s= \pm 4$ harmonics in the above example stems from the spin-2 nature of gravitational waves. The violations associated with the $s= \pm 4$ harmonics couple the \pm 2 -helicity waves, leading to changes of \pm 4 in helicity. Similarly, the $s= \pm 2$ harmonics arise for spin- 1 photons in the $\mathrm{SME},{ }^{4}$ and $s= \pm 1$ harmonics appear in the spherical expansions for spin- $\frac{1}{2}$ fermions. ${ }^{5,6}$

\section{Acknowledgments}

This work is supported in part by the United States National Science Foundation under grant PHY-1520570.

\section{References}

1. Data Tables for Lorentz and CPT Violation, V.A. Kostelecký and N. Russell, 2016 edition, arXiv:0801.0287v9.

2. D. Colladay and V.A. Kostelecký, Phys. Rev. D 55, 6760 (1997); Phys. Rev. D 58, 116002 (1998).

3. V.A. Kostelecký, Phys. Rev. D 69, 105009 (2004); Q.G. Bailey and V.A. Kostelecký, Phys. Rev. D 74, 045001 (2006); V.A. Kostelecký and J. Tasson, Phys. Rev. D 83, 016013 (2011).

4. V.A. Kostelecký and M. Mewes, Phys. Rev. Lett. 99, 011601 (2007); Ap. J. 689, L1 (2008); Phys. Rev. D 80, 015020 (2009); Phys. Rev. Lett. 110, 201601 (2013).

5. V.A. Kostelecký and M. Mewes, Phys. Rev. D 85, 096005 (2012).

6. V.A. Kostelecký and M. Mewes, Phys. Rev. D 88, 096006 (2013).

7. Q.G. Bailey, V.A. Kostelecký, and R. Xu, Phys. Rev. D 91, 022006 (2015).

8. V.A. Kostelecký and J.D. Tasson, Phys. Lett. B 749, 551 (2015).

9. V.A. Kostelecký and M. Mewes, Phys. Lett. B 757, 510 (2016).

10. F.W. Stecker, Astropart. Phys. 35, 95 (2011).

11. V. Vasileiou et al., Phys. Rev. D 87, 122001 (2013); F. Kislat and H. Krawczynski, Phys. Rev. D 92, 045016 (2015).

12. S.R. Parker et al., Phys. Rev. Lett. 106, 180401 (2011); M. Mewes, Phys. Rev. D 85, 116012 (2012); Y. Michimura et al., Phys. Rev. D 88, 111101 (2013); S.R. Parker et al., Phys. Lett. A 379, 2681 (2015).

13. J.S. Díaz, V.A. Kostelecký, and M. Mewes, Phys. Rev. D 89, 043005 (2014); F.W. Stecker et al., Phys. Rev. D 91, 045009 (2015).

14. A.H. Gomes, V.A. Kostelecký, and A.J. Vargas, Phys. Rev. D 90, 076009 (2014).

15. V.A. Kostelecký and A.J. Vargas, Phys. Rev. D 92, 056002 (2015).

16. C.-G. Shao et al., Phys. Rev. D 91, 102007 (2015); J.C. Long and V.A. Kostelecký, Phys. Rev. D 91, 092003 (2015); C.-G. Shao et al., arXiv:1607.06095.

17. R. Bluhm et al., Phys. Rev. Lett. 88, 090801 (2002); Phys. Rev. D 68, 125008 (2003); V.A. Kostelecký and M. Mewes, Phys. Rev. D 66, 056005 (2002). 